\title{
Fractal microgeometry of original and hydgrogen-charged graphene multilayer films surface: research by the low- temperature sorption of nitrogen method
}

\author{
Svetlana Vasilyeva ${ }^{1}$, Anna Zheltova $^{2}$, Alexey Merkushkin ${ }^{3}$, and Alexander Smolyanskiy ${ }^{1,3}$ \\ ${ }^{1}$ «Karpov Institute of Physical Chemistry», 105064 Moscow, Russian Federation. \\ ${ }^{2}$ Lomonosov Moscow State University, Faculty of Bioengineering and Bioinformatics, 119234 Moscow Russian Federation \\ ${ }^{3}$ Dmitry Mendeleev University of Chemical Technology of Russia, 125047 Moscow, Russian Federation
}

\begin{abstract}
Changes in the surface microgeometry of multilayer graphene films, as a result of hydrogen adsorption ( $1 \%$ mass.), have been examined by the method of low-temperature fractal sorption of nitrogen. It is established that hydrogen atmosphere treatment leads to the significant development of graphene surface and lowers the first layer heat adsorption from 289 down to $260 \mathrm{~J} / \mathrm{kg}$. There have been found out as well the overall mesopores concentration growth and pore size distribution function broadening. At that, the surface fractal dimension value has grown from 2,52 up to 2,67, which is to give us an evidence of the surface chaotization as a result of hydrogen withdrawal into the atmosphere and the graphene surface erosion.
\end{abstract}

\section{Introduction}

The use of water purification plants based on the ozone electrochemical generators necessitates the utilization of hydrogen evolved on the electrolyzer cathode [1]. The multilayer graphene films are considered to be an advanced sorbent for the hydrogen absorption [2]. It is commonly known $[2,3,10,11]$ that the hydrogen absorption on graphene is a complex physicochemical process, which, in particular, leads to some substantial changes in the film surface microgeometry. However, the conclusions [3], being based on the results of roughness measurement of the original and hydrogencharged graphene films surface (hereinafter $-\mathrm{G}$ and $\mathrm{GH}$ respectively) obtained by the atomic force microscopy (AFM) method, require an additional experimental proof which is achievable through the sorption methods $[4,5]$ use. An advantage of such sorption methods is a possibility of an overall analysis of the surface of the test sample, whereas AFM-measurement provides only local surface analysis.

On the other hand [4], the polymolecular adsorption theory of Brunauer-Emmett-Teller (BET) is based on the presumption that the surface the adsorbate molecules are deposited on, is to be perfectly smooth. For the surface roughness recordkeeping, the fractal sorption approach [5] is applied.

Therefore, the use of the approach in question [5] is essential for the estimation of the $G$ surface changes caused by the hydrogen adsorption.

The purpose of the present research was to examine the G multilayer films surface, before and after treatment in the hydrogen atmosphere, using the low-temperature nitrogen sorption method; to analyze the obtained sorption data using the BET sorption isotherm and the fractal sorption equations [5].

\section{Experimental technique}

To prepare the graphene oxide using the method [6], $2 \mathrm{~g}$ of graphite powder, $105 \mathrm{ml}$ of sulphuric acid and $1 \mathrm{~g}$ $\mathrm{NaNO} 3$ were being stirred during 30 minutes at the ambient temperature. Then, $8 \mathrm{~g} \mathrm{KMnO} 4$ were gradually being added into the reaction mixture during 2 hours, keeping the reaction mixture stirred in the ice bath.

After the stirring within one hour at $35^{\circ} \mathrm{C}, 200 \mathrm{ml}$ of distilled water were been added slowly to the reaction mixture. Then, the temperature of the reaction mixture was gradually raised up to $98^{\circ} \mathrm{C}$ and the reaction went on within other 15 minutes. After the cooldown of the mixture to the ambient temperature, $150 \mathrm{ml}$ of distilled water containing $6 \mathrm{ml}$ of hydrogen peroxide was added thereto. After the process termination the reaction mixture was filtered, rinsed and redispersed in the 5\% $\mathrm{HCl}$ solution. Then, the graphene oxide powder was being washed with water until the neutral $\mathrm{pH}$ was reached and then was dehydrated in vacuum at $60^{\circ} \mathrm{C}$. The derived product was exposed to a flash drying at $1000^{\circ} \mathrm{C}$ during 12 minutes under control.

The produced $\mathrm{G}$ hydrogenation was carried out in the special device under various pressure conditions (up to 1 $\mathrm{MPa}(\sim 0,987 \mathrm{~atm}$.) at $77 \mathrm{~K}$ (Fig. 1).

It was found out that hydrogen sorption on $G$ could be adequately described by the Freundlich sorption isotherm equation [7]:

\footnotetext{
* Corresponding author: vasileva@nifhi.ru
} 


$$
\ln \mathrm{A}=\ln \mathrm{K}+1 / \mathrm{n} \ln \mathrm{P}
$$

where the regression parameters $\operatorname{lnK}=-6,294 \pm 0,157$, $1 / \mathrm{n}=0,696 \pm 0,019$, the correlation factor value is 0,994 (Fig. 1).

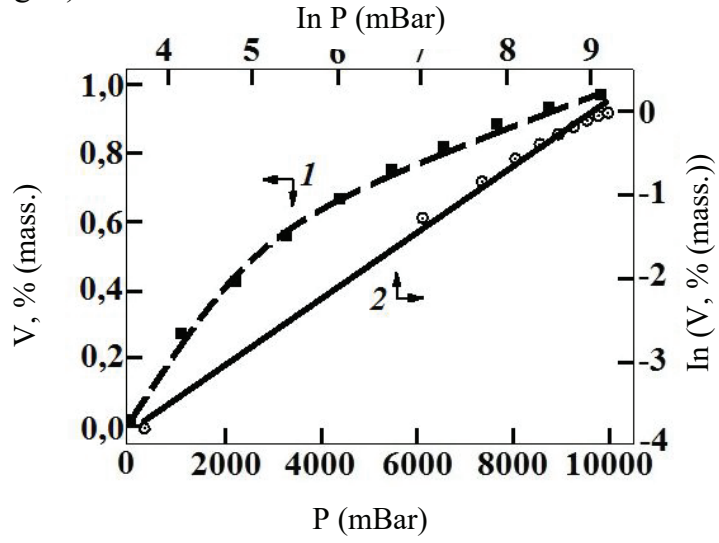

Fig. 1. Parameters of hydrogen sorption on graphene surface during hydrogenation process.

The Freundlich sorption isotherm is observable in case of adsorbates uptake in heterogeneous systems featured by the presence of the interactive active centers (AC) sorption heat exponential distribution $[4,7]$. The calculated parameter values of the Freundlich pattern the invaiables $\mathrm{K} \sim 1,85 \cdot 10-3 \quad$ (\% (mass.) $\mathrm{H} 2 / \mathrm{g}) 1-\mathrm{n}(\%$ (mass.) H2/g)n as well as power index $\mathrm{n} \sim 1,437$, - feature the graphene hydrogen absorption capacity and the sorption intensity. In the present research the $\mathrm{G}$ samples with the highest hydrogen absorption ( $1 \%$ (mass.)) were used.

The examination of low-temperature nitrogen sorption on $\mathrm{G}$ and $\mathrm{GH}$ samples was carried out at $77 \mathrm{~K}$ on the Quantachrome QuadraWin plant. The sorption experiment results interpretation in the context of the BET sorption isotherm equation was carried out using the proper software for the device - there were determined the values of the BET equation invaiables C- constant as well as monolayer capacity $\mathrm{Vm}, \mathrm{S}-$ surface area of G and GH (Table 1).

Table 1. Comparison of nitrogen sorption parameters of normal and hydrogenated graphene

\begin{tabular}{|c|c|c|}
\hline Name & $\mathrm{G}$ & $\mathrm{GH}$ \\
\hline $\mathrm{C}$ & 53,52 & 15,17 \\
\hline $\mathrm{Q}_{1}, \mathrm{~kJ} / \mathrm{kg}$ & 289,02 & 260,19 \\
\hline $\mathrm{S}, \mathrm{m}^{2} / \mathrm{g}$ & 342,8 & 1201,3 \\
\hline $\begin{array}{c}\mathrm{V}_{\mathrm{m}}, \\
\mathrm{cm}^{3} / \mathrm{g}\end{array}$ & 0,098 & 0,345 \\
\hline $\mathrm{B}$ & $-0,48 \pm 0,01$ & $-0,33 \pm 0,01$ \\
\hline$\Delta$ & $-0,4477$ & 0,0065 \\
\hline $\mathrm{D}_{\mathrm{s}}$ & 2,52 & 2,67 \\
\hline
\end{tabular}

\section{Results and Discussion}

The hydrogenization of $G$ leads to the substantial nitrogen sorption parameters change (Table 1): it was found triple $\mathrm{C}$-constant derate whereas $\mathrm{S}$ value grows up by a factor of 4 circa. It is known $[4,12,13,14,17]$, that $\mathrm{C}$-constant features an interaction between the adsorbate and $\mathrm{G}, \mathrm{GH}$ - surfaces as well as nitrogen molecules interaction:

$$
\mathrm{C}=\exp [((\mathrm{Q} 1-\lambda)) \mathrm{RT}]
$$

where Q1 - adsorption heat in the first layer, which is determined by the nitrogen molecules and G, GH-surface interaction, $\mathrm{kJ} / \mathrm{kg} ; \lambda$ - the nitrogen condensation heat (198 kJ/kg [8]); T - temperature, K; R - universal gas constant for nitrogen $(297 \mathrm{~J} /(\mathrm{kg} \bullet \mathrm{K})[8])$. As it follows from the Table 1, the hydrogen adsorption leads to the Q1 value decrease which proves the change of type of the nitrogen molecules and $\mathrm{G}$ interaction over adsorbed hydrogen.

Let us discuss the surface development effect in the context of the fractal sorption approach $[5,15,16,18]$. The point of the given approach is the Ds - surface fractal dimension determination:

$$
\ln \left(\mathrm{V} / \mathrm{V} \_\mathrm{m}\right)=\mathrm{A}+\mathrm{B} \ln \left[\ln \left(\mathrm{p} \_\mathrm{s} / \mathrm{p}\right)\right]
$$

where $\mathrm{V}$ - adsorbtion, cm3/g; A, B - regression factors; $\mathrm{p}$ - the nitrogen vapor pressure current value, mbar; $\mathrm{ps}$ the nitrogen saturated vapor pressure $(1940,44 \mathrm{mBar}$ at $77 \mathrm{~K}[8])$
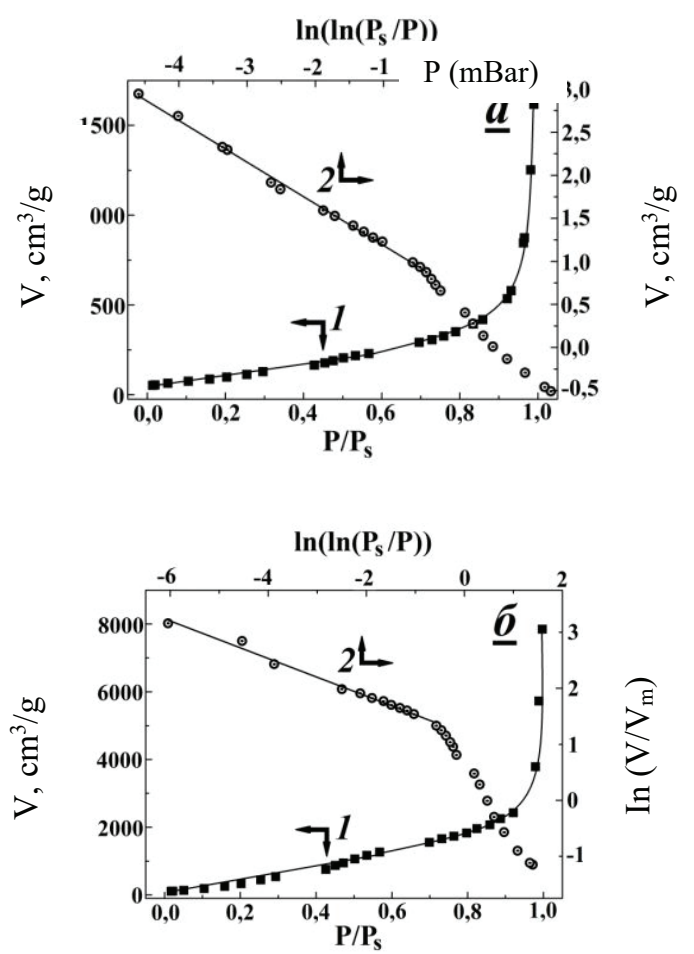

Fig. 2 (a, b). Determination of regression factor value for normal and hydrogenated graphene.

It is necessary to point out that $2<\mathrm{Ds}<3$, moreover, $\mathrm{Ds}=2$ corresponds to an xy-plane in Euclidean space and $\mathrm{Ds}=3$ - to a perfectly chaotic surface. The value B for $\mathrm{G}$, 
$\mathrm{GH}$ can be determined from the tilting angle of the straight-line portion of $\ln (\mathrm{V} / \mathrm{Vm})$ от $\ln [\ln (\mathrm{ps} / \mathrm{p})]$ functional connection(Fig. 2 (a, b), curve 2; Table1).

In accordance with [5], the second regression factor depends on Ds and on the adsorption mechanism (Van der Waals forces interaction or capillary condensation). In case of Van der Waals interaction mechanism

$$
\mathrm{B}=\left(\mathrm{D} \_\mathrm{s}-3\right) / 3
$$

In case of capillary condensation mechanism:

$$
\mathrm{B}=\mathrm{D} \_\mathrm{s}-3
$$

In case of minor coverage of surface the adsorbate adsorbent interaction mechanism choice is to be done with the following criteria:

$$
\delta=3(1+\mathrm{B})-2
$$

The results of the parameter $\delta$ calculations are shown in the Table 1. In accordance with [5], for $G$, the negative $\delta$ value says for capillary condensation mechanism prevalence by the nitrogen adsorption on graphene. In this case the $\mathrm{G}$ surface fractal dimension can be determined according to the formula (5) (Table 1).

For GH $\delta \geq 0$ this fact means that the hydrogen modification of the graphene surface can lead to the nitrogen-GH surface interaction Van der Waals mechanism realization. The surface fractal dimension value $(2,0069)$ calculated according to the formula (4) is slightly different from the critical value $\mathrm{Ds}=2$, which corresponds to the smooth surface. In view of this one can presume that the graphene surface hydrogenization can lead to a significant reduction of surface roughness.

However, this conclusion is in controversy with the abovementioned evidence of the hydrogen-charged graphene surface area $\mathrm{S}$ increase as well as with the results of the pore size distribution function calculation (PDF) for G and GH, shown in the Fig. 3.

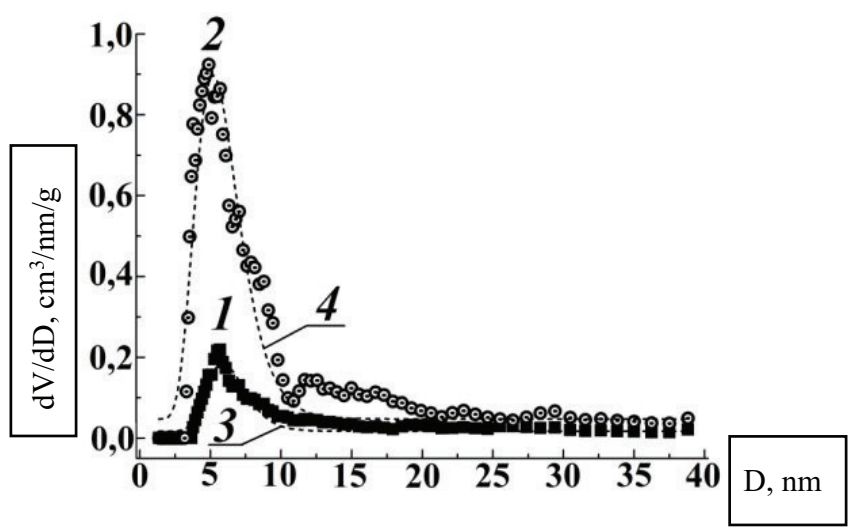

Fig. 3. Results of the pore size distribution function calculation for normal and hydrogenated graphene.

As it follows from the Fig. 3, hydrogenization leads to the significant PDF extension as well as to the pore concentration growth in the highest PDF $(5-7 \mathrm{~nm})$ by the factor of 5 in comparison with the original graphene PDF. Furthermore, PDF of GH contains two new wide peaks of low intensity at $\sim 10$ and $15 \mathrm{~nm}$. These peaks appearance may display the coalescence processes for the pores of the basic highest size $(5-7 \mathrm{~nm})$. It is necessary to notice as well that in both cases the PDF form could be adequately described using the lognormal distribution method (Fig. 3, curves 3, 4).

Therefore, the aggregate of the presented facts, namely, increase of $\mathrm{S}$ and significant broadening of PDF in case of $\mathrm{GH}$, is in controversy with the conclusion about the surface roughness reduction trend. Furthermore, determination of the $\delta$-parameter has been done inaccurately, - taking into consideration the lower variation range limit in the B-parameter value, inserting into (6) the condition $\delta<0$ which means the interaction mechanism is determined as capillary condensation. Then the surface fractal dimension calculation according to the equation (5) leads to the value of $\mathrm{Ds}=2,67$, that says for the hydrogen-charged graphene surface development.

\section{Conclusions}

The ambiguousness in the determination of the nitrogen molecules - hydrogen-charged graphene surface interaction revealed in the present research, may prove the fact that the hydrogen sorption process is the complex one and comes with graphene surface erosion due to dehydrogenation $[9,19,20]$. It can be presumed that formation of new nano-/micropores on the hydrogenated graphene surface takes place as the result both of such erosion and hydrogen evolution. The subsequent nitrogen adsorption allows fixing the changes on the surface that have taken place. The Van der Waals interaction realization during the nitrogen molecules sorption on the hydrogen-charged graphene surface can be explained by the fact that nano- and micropores of the nitrogen molecules occurring on the adsorbent surface are full of hydrogen molecules which excludes any possibility of capillary condensation mechanism realization. Reduction of the sorption heat value in the first layer (table 1) can as well favor such hypothesis.

The further experimentation of the original and hydrogen-charged graphene surface using scanning electron microscopy (SEM) and AFM in order to prove (or disprove) the results shown in the present article, is evident. The results of multifractal analysis of SEMand AFM-pictures of the G-and GH-surfaces will be stated in the following article.

The present research was supported by the Ministry of Education and Science of the Russian Federation (Grant of the 20th of October, 2014, № 14.576.21.0053; The Applied Research Unique Identifier RFMEFI57614X0053). The authors hereof would like to thank Mr. Zahra Gohari Bajestani (Sabancı Üniversitesi, İstanbul, Turkey) for the original and hydrogen-charged graphene samples production.

\section{References}

1. G.Ph. Potapova, M.I. Ikim, S.A. Korneychuk, A.S. Smolyanskiy, Composites and nanostructures 5, 23 $32(2013)$ 
2. Yu.S. Nechaev, T.N. Veziroglu, Int. J. Chem. 7, $207-212$ (2015)

3. S. Goler, C. Coletti, V. Tozzini, V. Piazza, T. Mashoff, F. Beltram, V. Pellegrini, S. Heun, J. Phys. Chem. 117, 11506-11513 (2013)

4. S. Greg, K. Sing, Adsorption, specific surface, porosity, 306 (1984)

5. M.K. Wu, Aerosol Sci. and Technol. 25, 392-398, (1996)

6. W.S. Hummers, R.E. Offeman, J. Amer. Chem. Soc. 80, 1339 (1958)

7. H.M.F. Freundlich, J. Phys. Chem. A57, 385 (1906)

8. M.B. Vargaftik, Reference book for thermal-physical properties of gases and liquids, 720, (1972)

9. A. Thangaraja, S.M. Shinde, K. Golap, R. Papon, S. Sharma, R. Vishwakarma, K.P. Sharma, M. Tanemura Appl. Phys. Lett., 106, 253106 (2015)

10. C. Wonbong, Graphene: synthesis and applications, 35-36, (2012).

11. D. Dreyer, S. Park, C. Bielawski, R.S. Ruoff, Chem. Soc. Rev., 39, 228-240, (2010)

12. Z. M. Ao, Q. Jiang, R. Q. Zhang, T. T. Tan, S. Li, J. Appl. Phys., (2009)

13. O. Leenaerts, B. Partoens, and F. M. Peeters, Phys. Rev. B 80, 245422, (2009)

14. P. Ruffieux et al., Phys. Rev. B 66, 245416 (2002).

15. S. Ross and J. Olivier, On Physical Adsorption, (1964)

16. W. Choi, I. Lahiri, R. Seelaboyina, Y.S. Kang, Crit. Rev. Solid State Mater. Sci., 35, 52, (2010)

17. S. Gilje, S. Han, M. Wang, K. L. Wang, R. B. Kaner, Nano Lett. 7, 3394 (2007)

18. M.R. Falvo, G.J. Clary, M. Taylor II, V. Chi, F.P. Brooks Jr., S. Washburn, R. Superfinem, Nature, 389, 582, (1997)

19. S. Bae et al., Nat. Nanotechnol. 5, 574-578, (2010)

20. A. W. Tsen et al., Science 336, 1143-1146 (2012) 